\title{
Immunohistochemical Evaluation of Ki-67 and Comparison with Clinicopathologic Factors in Breast Carcinomas
}

\author{
Nguiessan Alphonse Aman ${ }^{1 *}$, Brahima Doukoure ${ }^{2}$, Kouadio Donatien Koffi ${ }^{1}$, \\ Baumaney Sylvanus Koui ${ }^{2}$, Zie Cheick Traore ${ }^{1}$, Mohamed Kouyate ${ }^{2}$, Ibrahiman \\ Toure $^{1}$, Ahoua Benjamin Effi ${ }^{1}$
}

\begin{abstract}
Background: Patients primarily received tamoxifen based on their menopausal status due to the lack of immunohistochemistry. A recent study has shown that hormonal receptors were not correlated with menopausal status, and thus, indicating that they present limited therapeutic and prognostic significance in breast cancer management. This study aimed to evaluate Ki-67 value and analyze its association with clinicopathologic parameters in breast cancer patients. Methods: The formalin-fixed paraffin-embedded breast tissue blocks of 125 patients with primary breast carcinomas were subjected to immunohistochemical analysis using Ventana Benchmark ${ }^{\circledR}$ GX automated immunostainer. Analysis of variance and Chi-2 test were used to examine the relationship between $\mathrm{Ki}-67$ and clinicopathologic variables. Results: The mean age of 125 patients included in the study was 47.7 years. The average score of Ki-67 was $56.0 \%$. 84.8\% of patients showed Ki-67 $\geq 14 \%$. Mean scores of Ki-67 were correlated with grade $(p=0.006)$, PR $(p=0.026)$, histological type, ER, combined ER/RP, and molecular subtype $(p<0.001)$. Ki-67 was independent of HER2 $(p=0.402)$ and menopausal status $(p=0.471)$. The frequency of Ki-67 according to St Gallen 2011 was associated with histological type $(p=0.005)$, grade $(p=0.005)$, ER $(p<0.001)$, combined ER/PR $(p=0.004)$, and molecular subtype $(p=0.004)$. There was no significant relationship between the distribution of Ki-67 and the age of the patients $(p=0.859)$, menopausal status $(p=0.979)$, PR $(p=0.149)$, and HER2 $(p=0.597)$. Conclusion: Ki-67 is useful for treatment decisions in primary breast cancer patients. The high value of Ki-67 was associated with adverse clinicopathologic factors. The increased Ki-67 value should be carefully investigated in triple negative patients.
\end{abstract}

Keywords: ER- HER2- Ki-67- PR- primary breast cancer

Asian Pac J Cancer Prev, 20 (1), 73-79

\section{Introduction}

Breast cancer is the most common cancer in women worldwide (Ferlay et al., 2012; Ferlay et al., 2013). The incidence of breast cancer is increasing in sub-Saharan Africa, where it is the most frequent leading cause of death in women (Ferlay et al., 2012; Ferlay et al., 2013). Breast tumor malignancy is the most prevalent cancer in women in Ivory Coast followed by cervical cancer according to the Cancer Registry of Abidjan (Ferlay et al., 2013). Several classic breast carcinoma markers, such as estrogen receptor (ER), progesterone receptor (PR), Ki-67 proliferative index, and human epidermal growth factor of receptor 2 (HER2) are relevant for therapy strategy and prognosis (Perou et al., 2000; Sorlie et al., 2001; Cheang et al., 2009; Cuzick et al., 2011). Ki-67 antigen, described by Gerdes (1983), is a marker of proliferation controlling the cell cycle. The Immunohistochemistry (IHC) analysis of Ki-67 is the reference test to assess tumor cell proliferation in breast tissue embedded in paraffin according to the International Ki-67 assessment in Breast Cancer Working Group (Dowsett et al., 2011). This marker presents predictive and prognostic value in clinical management of breast cancer (Stuart-Harris et al., 2008; Fasching et al., 2011; Denkert et al., 2013). Although the IHC determination of Ki-67 is integrated into routine clinical practice, its positive cut-off level remains controversial (Cheang et al., 2009; Fasching et al., 2011; Aleskandarany et al., 2011; Goldhirsch et al. 2011; Polley et al., 2013). Prior to the advent of IHC in Ivory Coast in 2013, breast cancer patient care was primary based on chemotherapy and hormonotherapy. The eligible criterion for hormonotherapy was based on patients' menopausal status due to the lack of routine IHC testing of ER, PR, HER2, and Ki-67. Studies done by Effi et al., (2017) have shown that assumption should be considered since they clarified that RH status was not correlated with menopausal status. Hereby, the menopausal status 
and the hormonal receptor (HR) status present limited prognostic and therapeutic significance in clinical care of the breast cancer patients. Today, IHC examination of Ki-67 with ER, PR, HER2 identifies five molecular breast cancer subtypes for effective and adequate personalized therapy (Perou et al., 2000; Sorlie et al., 2001; Cheang et al., 2009). However, no studies have evaluated the prevalence, distribution and, theranostic implications of Ki-67 proliferative index in breast cancer patients. The purpose of this study was to assess the value of Ki-67 and its association with menopausal status, Nottingham grade, histological type, expression levels of ER, PR, and HER2 to guide the therapeutic choice of breast cancer patients.

\section{Materials and Methods}

\section{Patients}

The prospective study was conducted between October 2014 and June 2015, including 125 patients diagnosed with primary invasive breast carcinomas at the Central Laboratory in Abidjan, Ivory Coast. The histologic diagnosis was performed upon paraffin-embedded breast tissue blocks sampled from $111(88.8 \%)$ core needle biopsies and $14(11.2 \%)$ surgical samples. On each sample, the histologic type and the Nottingham grade were established according to the WHO Classification of breast tumors (Lakhani et al., 2012) and the criteria of Elston and Ellis (1991), respectively. The studied variables were classic clinicopathologic parameters (age, menopausal status, histological type, and tumor grade) and hormonal receptor status (ER, PR, combined ER/PR), Ki-67, and HER2. Paraffin-embedded blocks of breast tissue were subjected to the IHC testing.

\section{Immunohistochemical analysis}

The immunohistochemical analysis was performed on $3 \mu \mathrm{m}$ thickness of breast tissue sections. Tissue sections were deparaffinized and heated in the drying oven BINDER $^{\circledR}$ (BINDER Company, Tuttlingen, Germany) for at least $12 \mathrm{~h}$ at $600 \mathrm{C}$ to unmask the antigenic sites. The sections were stained using the Ventana BenchMark ${ }^{\circledR} \mathrm{GX}$ in automatic mode (Ventana Medical Systems Inc., Tucson, AZ, USA) for the assessment of ER, PR, HER2, and Ki67. The monoclonal antibody clones, developed in rats, consisted of SP1 for the ER, 1E2 for the PR, 4B5 for the HER2, and 30-9 for the Ki-67.

\section{Immunostaining assessment}

The semi-quantitative analysis was applied to the scoring systems of ER, PR, HER2, and Ki-67. The scoring of ER and PR was based on the staining intensity (weak, moderate, intense) and the percentage of tumor cells showing a nuclear immunostaining for ER and PR (range: 0-100\%). Breast tissue sections were considered positive for ER and $P R$ if $\geq 1 \%$ of tumor cells, which displayed a positive nuclear staining (Hammond et al., 2010). Ki-67 score was expressed as the percentage of the number of immunostained nuclei among the total number of nuclei of tumor cells regardless of the immunostaining intensity. The counting was performed in three randomly selected fields of the breast tissue section at $\mathrm{x} 400$ magnification. When the staining was homogeneous, the counting was carried out at two peripheral and one central fields. The grading of hot spots was considered in case of heterogeneous tumor (Dowsett et al., 2011). Ki-67 score ranged from 0 to $100 \%$ and its positive cut-off level was $\geq 14 \%$ according to Cheang et al., (2009). The expression of proliferation index Ki-67 is categorized into 3 groups: low (Ki-67 $\leq 15 \%)$, intermediate (Ki-67:16-30\%), and high (Ki-67>30\%) according to the recommendations of the St Gallen International Consensus of Experts (Goldhirsch et al., 2009; Goldhirsch et al., 2011). The evaluation criteria of HER 2 status were based on the intensity of cell membrane immunostaining and the percentage of membrane positive cells, giving a score range of 0-3+ (Wolff et al., 2013). HER2 negative (score 0 or $1+$ ) was decided when no staining was observed or membrane staining in less than $10 \%$ of tumor cells or a faint partial membrane staining in more than $10 \%$ of tumor cells, HER2 equivocal (score 2+) when a weak to moderate complete membrane staining in more than $10 \%$ of tumor cells and HER 2 positive (score $3+$ ) when a strong complete membrane staining in more than $10 \%$ of tumor cells. HER 2 equivocal was not evaluated in our study due to the lack of fluorescence in situ hybridization.

The IHC scoring of ER, PR, HER2, and Ki-67 was reviewed independently by two pathologists. For the purpose of this study, HER2 equivocal and IHC intensity for ER and PR were not considered.

\section{Statistical analysis}

Data were collected in an Excel database from Windows 8 (Microsoft Corporation, Redmond, WA, USA) and analysed in SPSS 20.0. The analysis of variance (ANOVA) evaluated the relationship between the mean score of $\mathrm{Ki}-67$ as a continuous variable with clinicopathologic factors, including menopausal status, histological type and Nottingham grade, ER, PR, HER2, combined ER/PR, and molecular subtype. The ChiSquare Test analyzed the correlation between Ki-67 as a categorical variable with clinicopathologic parameters. A probability p-value $<0.05$ was considered statistically significant. The data reported as means \pm standard deviation for ANOVA and frequencies for Chi-Square Test.

\section{Results}

\section{Characteristics of patients}

The average age of all patients was $47.7 \pm 10.8$ years (extremes: $29-83$ years). Among the 125 patients, $59.2 \%$ were premenopausal compared to $40.8 \%$ of postmenopausal. Invasive ductal carcinoma of no specific type (IDC-NST) $(80.8 \%)$ was predominant. Grade II tumors were $61.1 \%$ followed by grade III $(23.0 \%)$. The positivity of ER, PR, and HER2 were $54.0 \%, 46.7 \%$, and $13.7 \%$, respectively. ER/PR+ HER2- breast carcinomas $(50.3 \%)$ were prevalent followed by triple negative subtype $(36.8 \%)$ and HER2+ subtype $(13.2 \%)$. The patients clinicopathologic factors are shown in the Table 1. 
DOI:10.31557/APJCP.2019.20.1.73

Comparison of Ki-67 with Pathologic Factors in Breast Cancer

Table 1. Clinicopathologic Characteristics in the Breast Carcinoma Patients

\begin{tabular}{|c|c|}
\hline Variables & Number of patients $\mathrm{N}=125$ \\
\hline \multicolumn{2}{|l|}{ Age (years) } \\
\hline Mean \pm SD & $47.7 \pm 10.8$ \\
\hline Extremes & $29-83$ \\
\hline \multicolumn{2}{|l|}{ Menopausal status } \\
\hline$<50$ years & 74 \\
\hline$\geq 50$ years & 51 \\
\hline \multicolumn{2}{|l|}{ Histological type } \\
\hline IDC-NST & 101 \\
\hline Lobular & 4 \\
\hline Other & 20 \\
\hline \multicolumn{2}{|l|}{ Nottingham grade } \\
\hline I & 18 \\
\hline II & 69 \\
\hline III & 26 \\
\hline undetermined & 12 \\
\hline \multicolumn{2}{|l|}{ Estrogen receptor } \\
\hline Positive & 66 \\
\hline Negative & 57 \\
\hline Uninterpretable & 2 \\
\hline \multicolumn{2}{|l|}{ Progesterone receptor } \\
\hline Positive & 57 \\
\hline Negative & 65 \\
\hline Uninterpretable & 3 \\
\hline \multicolumn{2}{|l|}{ HER2 } \\
\hline Positive & 16 \\
\hline Negative & 101 \\
\hline Equivocal & 3 \\
\hline Uninterpretable & 5 \\
\hline \multicolumn{2}{|l|}{ Hormonal receptor } \\
\hline $\mathrm{RO}+\mathrm{RP}+$ & 51 \\
\hline RO+RP- & 14 \\
\hline RO-RP+ & 5 \\
\hline RO-RP- & 50 \\
\hline \multicolumn{2}{|l|}{$\mathrm{Ki}-67$} \\
\hline Negative $(<14 \%)$ & 19 \\
\hline Positive ( $\geq 14 \%$ ) & 106 \\
\hline \multicolumn{2}{|l|}{ Molecular subtypes } \\
\hline Luminal A & 14 \\
\hline Luminal B/HER2- & 43 \\
\hline Luminal B/HER2+ & 11 \\
\hline $\begin{array}{l}\text { Non-luminal B/ } \\
\text { HER2+ }\end{array}$ & 4 \\
\hline Triple negative & 42 \\
\hline Unclassifiable & 11 \\
\hline
\end{tabular}

Distribution of Ki-67 mean score and clinicopathologic parameters

The average score of $\mathrm{Ki}-67$ in all patients was $56.0 \%$. Ki-67 was positively expressed in $84.8 \%$ of patients. The average score of $\mathrm{Ki}-67$ was $57.8 \%$ in premenopausal
Table 2. Relationship between Ki-67 Mean Score and Clinicopathologic Factors in 125 Patients

\begin{tabular}{|c|c|c|c|}
\hline Variables & Total (n\%) & $\begin{array}{c}\text { Ki-67 mean } \\
\text { score }(\% \pm \mathrm{SD})\end{array}$ & $\mathrm{p}$ \\
\hline Mean age \pm SD (years) & $47.7 \pm 10.8$ & $56.0 \pm 32.6$ & \\
\hline Menopausal status & & & 0.471 \\
\hline$<50$ years & $74(59.2)$ & $57.8 \pm 33.9$ & \\
\hline$\geq 50$ years & $51(40.8)$ & $53.5 \pm 30.8$ & \\
\hline Histological type & & & $<0.001 *$ \\
\hline IDC-NST & $101(80.8)$ & $58.9 \pm 31.9$ & \\
\hline Lobular & $4(3.2)$ & $94.5 \pm 6.7$ & \\
\hline Other & $20(16.0)$ & $31.5 \pm 22.9$ & \\
\hline Nottingham grade & & & $0.006^{*}$ \\
\hline I & $18(15.9)$ & $40.3 \pm 31.5$ & \\
\hline II & $69(61.1)$ & $56.58 \pm 33.8$ & \\
\hline III & $26(23.0)$ & $71.2 \pm 21.4$ & \\
\hline Estrogen receptor & & & $<0.001 *$ \\
\hline Positive & $66(54.0)$ & $45.1 \pm 31.8$ & \\
\hline Negative & $57(46.3)$ & $68.7 \pm 28.4$ & \\
\hline Progesterone receptor & & & $0.026^{*}$ \\
\hline Positif & $57(46.7)$ & $48.9 \pm 32.1$ & \\
\hline Négatif & $65(53.3)$ & $62.0 \pm 31.9$ & \\
\hline HER2 & & & 0.402 \\
\hline Positive & $16(13.7)$ & $50.5 \pm 23.5$ & \\
\hline Negative & $101(86.3)$ & $57.7 \pm 32.9$ & \\
\hline Ki-67 & & & $<0.001 *$ \\
\hline Low & $19(15.2 \%)$ & $5 \pm 3.8$ & \\
\hline Intermediate & $18(14.4 \%)$ & $24.5 \pm 5.0$ & \\
\hline High & $88(70.4 \%)$ & $73.5 \pm 20.6$ & \\
\hline Hormonal receptor & & & $<0.001 *$ \\
\hline $\mathrm{ER}+\mathrm{PR}+$ & $51(42.5)$ & $46.3 \pm 31.5$ & \\
\hline $\mathrm{ER}+\mathrm{PR}-$ & $14(11.7)$ & $37.2 \pm 31.3$ & \\
\hline ER-PR+ & $5(4.2)$ & $82.6 \pm 11.1$ & \\
\hline ER-PR- & $50(41.7)$ & $68.2 \pm 28.8$ & \\
\hline Molecular subtypes & & & $<0.001 *$ \\
\hline Luminal A & $14(12.3)$ & $6 \pm 3.8$ & \\
\hline Luminal B/HER2- & $43(37.7)$ & $64.4 \pm 26.4$ & \\
\hline Luminal B/HER2+ & $11(9.7)$ & $53.3 \pm 25.6$ & \\
\hline Non luminal B/HER2+ & $4(3.5)$ & $51.3 \pm 13.1$ & \\
\hline Triple negative & $42(36.8)$ & $70.6 \pm 27.8$ & \\
\hline
\end{tabular}

patients compared to $53.5 \%$ in post-menopausal women. There was a significant difference between the rates of $\mathrm{Ki}-67$ and the histological type. Ki-67 mean score was $40.3 \%, 56.58$, and $71.2 \%$ in Nottingham grade I, II, III, respectively. The ER+ (45.1\%), PR+ (48.9\%), and $\mathrm{ER} / \mathrm{PR}+(47.1 \%)$ patients had similarities as well as ER- (68.7\%), PR- (62.0\%), ER/PR- (68.2\%) individuals. HER2+ patients had Ki-67 average score of $50.5 \%$ compared with $57.7 \%$ of HER2- women. The average rate of $\mathrm{Ki}-67(70.6 \%)$ was the highest in the triple negative women while it was the lowest in the luminal A group (6\%) (Table 2). The average score of Ki-67 is significantly correlated with histological type, Nottingham grade, 
Table 3. Distribution of Frequency of Ki-67 According to St Gallen with Clinicopathologic Factors

\begin{tabular}{|c|c|c|c|c|}
\hline \multirow[b]{2}{*}{ Variables } & \multicolumn{3}{|c|}{ Expression of Ki-67 according to St Gallen } & \multirow[b]{2}{*}{$\mathrm{p}$} \\
\hline & $\leq 15 \%$ & $16-30 \%$ & $>30 \%$ & \\
\hline Mean age \pm SD (years) & $48.9 \pm 13.2$ & $47.2 \pm 11.6$ & $47.7 \pm 10.2$ & 0.859 \\
\hline Menopausal status & & & & 0.979 \\
\hline$<50$ years & $11(57.9)$ & $11(61.1)$ & $52(59.1)$ & \\
\hline$\geq 50$ years & $8(42.1)$ & $7(38.9)$ & $36(40.9)$ & \\
\hline Histological type & & & & $0.005^{*}$ \\
\hline IDC-NST & $14(73.7)$ & $10(55.6)$ & $77(87.5)$ & \\
\hline Lobular and Other & $5(26.3)$ & $8(44.4)$ & $11(12.5)$ & \\
\hline Grade & & & & $0.005^{*}$ \\
\hline I & $5(33.3)$ & $5(31.3)$ & $8(9.8)$ & \\
\hline II & $10(67.0)$ & $10(62.5)$ & $49(59.8)$ & \\
\hline III & $0(0.0)$ & $1(6.3)$ & $25(30.5)$ & \\
\hline Estrogen receptor & & & & $<0.0001 *$ \\
\hline Positive & $16(88.9)$ & $13(72.2)$ & $37(42.5)$ & \\
\hline Negative & $2(11.1)$ & $5(27.8)$ & $50(57.5)$ & \\
\hline Progesterone receptor & & & & 0.149 \\
\hline Positive & $13(65.0)$ & $9(50.0)$ & $35(41.2)$ & \\
\hline Negative & $7(35.0)$ & $9(50.0)$ & $50(57.5)$ & \\
\hline HER2 status & & & & 0.597 \\
\hline Positive & $1(6.3)$ & $2(11.8)$ & $13(15.5)$ & \\
\hline Negative & $15(93.7)$ & $15(88.2)$ & $71(84.5)$ & \\
\hline Hormonal receptor & & & & $0.004^{*}$ \\
\hline $\mathrm{ER}+\mathrm{PR}+$ & $12(66.7)$ & $9(52.9)$ & $30(35.3)$ & \\
\hline $\mathrm{ER}+\mathrm{PR}-$ & $4(22.2)$ & $4(23.5)$ & $6(7.1)$ & \\
\hline ER-PR+ & $0(0.0)$ & $0(0)$ & $5(5.9)$ & \\
\hline ER-PR- & $2(11.1)$ & $4(23.5)$ & $44(51.8)$ & \\
\hline Molecular subtype & & & & $0.004^{*}$ \\
\hline RO/RP+ HER2- & $14(87.4)$ & $11(68.8)$ & $32(39.0)$ & \\
\hline HER2 positive & $1(6.3)$ & $1(6.2)$ & $13(15.9)$ & \\
\hline Triple negative & $1(6.3)$ & $4(25.0)$ & $37(45.1)$ & \\
\hline
\end{tabular}

ER/PR+HER2-, luminal A and luminal B/HER2-; HER2 positive, luminal B/HER2+ and non luminal B/HER2+; SD, standard deviation; $p$, Probability p-value; $(*)$, statistically significant difference with $\mathrm{p}<0.05$.

ER, PR, and ER/PR status, and molecular subtype. No correlation was found between average value of $\mathrm{Ki}-67$ and menopausal status, and HER2 status (Table 2).

Distribution of Ki-67 expression according to St Gallen 2011 and clinicopathologic parameters

Premenopausal patients showed high Ki-67 (59.1\%) and intermediate $\mathrm{Ki}-67$ (61.1\%). Ki-67 value was high in IDC-NST (87.5\%). Low Ki-67 (33.3\%) and intermediate Ki-67 (31.3\%) were prevalent in grade I tumors. Grade II tumors were characterized by a trend of high Ki-67 in the 3 categories. Unlike grade I tumors, almost all grade III breast cancer patients had a high $\mathrm{Ki}-67$, and none of them presented low Ki-67. The frequency of low and intermediate $\mathrm{Ki}-67$ predominated in HR positive patients. Conversely, HR negative patients had a proportion of high Ki-67. The HER2- patients showed a less distant trend in three different groups of $\mathrm{Ki}-67$, while the proportions of intermediate $(11.8 \%)$ and high Ki-67 (15.5\%) were observed in HER2+ breast cancer patients (Table 3). The correlations between the frequency of Ki-67, as a categorical variable, with clinicopathologic parameters were similar to those of Ki-67 mean score, as a continuous variable, with these factors except categories of Ki-67 and PR (Tables 2 and 3).

\section{Discussion}

The evaluation of Ki-67 proliferative index is the first study done on the distribution of this marker in women with breast cancer in Ivory Coast. Interestingly, treatment of this cancer was based on ER, PR, and HER2 status after the advent of IHC in June 2013. The determination of Ki-67 and its relationship with clinicopathologic parameters have prognostic and predictive relevance (Sorlie et al., 2001; Cuzick et al., 2011; Fasching et al., 2011; Denkert et al., 2013). The average score of Ki-67 $(56.0 \%)$ of the study population, whose mean age was 47.7 
years, is different from that of several studies which have reported a rate varying from 20\%-34.9\% (Ermiah et al., 2012; Haroon et al., 2013; Ingolf et al., 2014; Wang et al., 2014; Tashima et al., 2015). The high mean score of Ki-67 in young women suggests the aggressive feature of breast cancer as shown by literature data (Haroon et al., 2013; Nishimura et al., 2014). This high value of Ki-67 could be due to the small sample size of our study population and the anti-Ki-67 antibody type. In fact, various studies have found an average score of Ki-67 which was $26.91 \%$ in 194 patients in Pakistan (Haroon et al., 2013), 29.6\% in 100 patients in Libya (Ermiah et al., 2012), and $31.22 \%$ in 1,259 patients in China (Sun et al., 2015). In this current study, the IHC analysis of Ki-67 antigen used clone antibody 30-9 from Ventana in breast cancer. In contrast, other studies used a variety of antibodies such as MM1, SP6, Ki-67-S5 clones (Polley et al., 2013) and human monoclonal clone antibody MIB1 from DAKO (Dowsett et al., 2011), which is recommended. The diverse use of anti-Ki-67 antibodies in several studies could explain the significant variability of Ki-67 mean score. In addition, the evaluation of Ki-67 was carried out in the densely marked zones named hot spots in heterogeneous breast carcinomas whose number was high in our study. These hot spots were considered compared to the recommended general average score (Dowsett et al., 2011) since they have been shown to be closely associated with the survival of patients (Honma et al., 2015; Arima et al., 2016). This study identified that the expression of $\mathrm{Ki}-67$, as a continuous variable, was correlated with histological type, tumor grade, molecular subtype, and ER, PR, and combined ER/PR status. However, no correlation was observed between Ki-67 as, a categorical variable, and PR status, HER2 status, age, and menopausal status. The correlation of Ki-67 with clinicopathologic factors varied from one study to another, although the purpose of its investigation was similar. Objectively, Ki-67 is relevant for assessing the prognosis of patients (Stuart-Harris et al., 2008; Cuzick al., 2011; Denkert et al., 2013), distinguishing luminal subtypes A and B (Cheang et al., 2009; Goldhirsh et al., 2011), and predicting therapeutic response (Fasching et al., 2011; Denkert et al., 2013). Undoubtedly, some authors have found relationships between Ki-67 expression and some clinicopathologic variables in a small sample size (Bouzubar et al.,1989; Ermiah et al., 2012; Haroon et al., 2013), while others have demonstrated strong associations of K-i67 with all clinicopathologic parameters in a large study population (Inwald et al., 2013; Park et al., 2012; Wang et al., 2014). As a result, the lack of correlation between Ki-67 and age, menopausal status, PR, and HER2 status could be due to the small size of our sample in this study. However, our study revealed that the expression level of Ki-67 is positively correlated with tumor grade. The more the tumor grade increases, the higher the Ki- 67 . Our result corroborates with several studies (Haroon et al., 2013; Inwald et al., 2013; Sun et al., 2015). In fact, the mitotic activity is one of the three major components of the breast carcinoma grading system according to the criteria of Elston and Ellis (1991). It reflects the intensity of cancerous cell proliferation as well as Ki-67 proliferative index which is present in different phases of the cell cycle except quiescent phase G0 (Gerdes et al., 1984). The higher Ki-67 is associated with high grade, the more aggressive the cancer is, and thus, jeopardizing the survival of patients with early recurrence and metastasis (Haroon et al., 2013; Inwald et al., 2013). In addition, Pinder et al., (1995) have shown that grade and Ki-67 appear to be complementary rather than equivalent prognostic factors. They have found a strong correlation between Ki-67 and grade in a multivariate study. When they excluded the grade from this multivariate analysis, the positivity of Ki-67 replaced it as the most important factor in predicting the survival of patients with breast cancer. Ki-67 proliferative index and Nottingham grade clarify the aggressiveness of breast cancer, and thus, confirming their prognostic significance (Reyal et al., 2013; Inwald et al., 2013). Our research study also showed significant differences between the frequency of different scores of Ki-67 and the expression levels of ER, PR, and HER2. The positivity of ER and PR was associated with low Ki-67, whereas HER2 overexpression was observed in patients with high Ki-67. Our results are consistent with those of various researchers (Aleskandarany et al., 2011; Inwald et al., 2013; Sun et al., 2015). Our data suggests that the more aggressive the cancer is (high value of $\mathrm{Ki}-67$ ), the lower the ER and PR expression levels or the higher the overexpressed HER2 is. Moreover, triple negative and HER2+ subtypes were associated with high score of Ki-67 compared with low Ki-67 in ER/PR+HER2- patients as confirmed by other studies (Wang et al., 2014; Sun et al., 2015). Our results indicate that triple negative and HER2+ women present worse prognosis than ER/PR+HER2patients. A careful study should be done to investigate the high value of Ki-67 in triple negative breast cancer patients. The strong correlation of Ki-67 with molecular subtypes also suggests that $\mathrm{Ki}-67$ helps predict response to neoadjuvant chemotherapy or hormonotherapy in patients in our study as described by Denkert et al., (2013) and Fasching et al., (2011).

The average of Ki-67 proliferative index is high. Ki-67 is correlated with histological type, tumor grade, and ER status. However, Ki-67 is independent of age, menopausal status, and HER2 status. This study indicates that IHC testing for Ki-67 with ER, PR, and HER2, giving the breast cancer molecular subtypes, is relevant for clinical utility in patients because of its prognostic and therapeutic implications. A thorough study should be done to examine the high value of Ki-67 in young women with triple negative breast cancer.

\section{Abreviations}

ER: Estrogen Receptor; PR: Progesterone Receptor, HER2: Human Epidermal Growth Factor Receptor 2; IHC: Immunohistochemistry; HR: Hormonal receptor; WHO: World Health Organization; ANOVA; Analysis of variance; IDC-NST: Invasive ductal carcinoma of no special type.

\section{Funding disclosures}

This work was funded by the Roche and Novartis Laboratories - Ivory Coast. These funders have no role in the study design, in the collection, analysis, 
and interpretation of data and in the preparation of the manuscript.

\section{Competing interests}

The authors have no conflict of interest.

\section{Acknowledgements}

The authors thank the Roche Laboratory and the Ministry of Health for implementing the first Unit of Immunohistochemistry at the Central Laboratory in Abidjan, Ivory Coast. The authors also thank Mr. Koffi Arthur and Ms. Gnenaho Pamela for performing the immunohistochemical technique.

\section{References}

Aleskandarany MA, Rakha EA, Macmillan RD, et al (2011). MIB1/Ki-67 labelling index can classify grade 2 breast cancer into two clinically distinct subgroups. Breast Cancer Res Treat, 127, 591-9.

Arima N, Nishimura R, Osako T, et al (2016). Comparison of the hot spot and the average cancer cell counting methods and the optimal cut off point of the Ki-67 index for luminal type breast cancer. Oncology, 90, 43-50.

Bouzubar N, Walker KJ, Griffiths K, et al (1989). Ki67 immunostaining in primary breast cancer: pathological and clinical associations. Br J Cancer, 59, 943-7.

Cheang MC, Chia SK, Voduc D, et al (2009). Ki67 index, HER2 status, and prognosis of patients with luminal $\mathrm{B}$ breast cancer. $J$ Natl Cancer Inst, 101, 736-50.

Cuzick J, Dowsett M, Pineda S, et al (2011). Prognostic value of a combined estrogen receptor, progesterone receptor, Ki-67, and human epidermal growth factor receptor 2 immunohistochemical score and comparison with the Genomic Health recurrence score in early breast cancer. J Clin Oncol, 29, 4273-8.

Denkert C, Loibl S, Müller BM, et al (2013). Ki67 levels as predictive and prognostic parameters in pretherapeutic breast cancer core biopsies: a translational investigation in the neoadjuvant GeparTrio trial. Ann Oncol, 24, 2786-93.

Dowsett M, Nielsen TO, A'Hern R, et al (2008). International Ki-67 in Breast Cancer Working Group. Assessment of Ki67 in breast cancer: recommendations from the International Ki67 in Breast Cancer working group. J Natl Cancer Inst, 103, 1656-64.

Effi AB, Aman NA, Koui BS, et al (2017). Immunohistochemical determination of estrogen and progesterone receptors in breast cancer: relationship with clinicopathologic factors in 302 patients in Ivory Coast. BMC Cancer, 17, 115.

Elston CW, Ellis IO (1991). Pathological prognostic factors in breast cancer. I. The value of histological grade in breast cancer: experience from a large study with long-term followup. Histopathology, 19, 403-10.

Ermiah E, Buhmeida A, Abdalla F, et al (2012). Prognostic value of proliferation markers: immunohistochemical Ki-67 expression and cytometric S-Phase Fraction of women with breast cancer in Libya. J Cancer, 3, 421-31.

Fasching PA, Heusinger K, Haeberle L, et al (2011). Ki67, chemotherapy response, and prognosis in breast cancer patients receiving neoadjuvant treatment. BMC Cancer, 11, 486.

Ferlay J, Soerjomataram I, Ervik M, et al (2013). GLOBOCAN 2012 v1.0, Cancer Incidence and Mortality Worldwide: IARC Cancer Base No. 11 [Internet]. Lyon, France:
International Agency for Research on Cancer; Available from: http://globocan.iarc.fr, accessed on 15/7/2015.

Ferlay J, Steliarova-Foucher E, Lortet-Tieulent J, et al (2012). Cancer incidence and mortality patterns in Europe: estimates for 40 countries in 2012. Eur J Cancer, 49, 1374-403.

Gerdes J, Schwab U, Lemke H, et al (1983). Production of a mouse monoclonal antibody reactive with a human nuclear antigen associated with cell proliferation. Int J Cancer, $\mathbf{3 1}$, 13-20.

Gerdes J, Lemke H, Baisch H, et al (1984). Cell cycle analysis of a cell proliferation-associated human nuclear antigen defined by the monoclonal antibody Ki-67. J Immunol, 133, 1710-15.

Goldhirsch A, Ingle JN, Gelber RD, et al (2009). Thresholds for therapies: highlights of the St Gallen International Expert Consensus on the primary therapy of early breast cancer 2009. Ann Oncol, 20, 1319-29

Goldhirsch A, Wood WC, Coates AS, et al (2011). Strategies for subtypes-dealing with the diversity of breast cancer: highlights of the St Gallen International Expert Consensus on the Primary Therapy of Early Breast Cancer 2011. Ann Oncol, 23, 2997-3006.

Hammond ME, Hayes DF, Dowsett M, et al (2010). American Society of Clinical Oncology/College of American Pathologists guideline recommendations for immunohistochemical testing of estrogen and progesterone receptors in breast cancer. $J$ Clin Oncol, 28, 2784-95.

Haroon S, Hashmi AA, Khurshid A, et al (2013). Ki67 index in breast cancer: correlation with other prognostic markers and potential in pakistani patients. Asian Pac J Cancer Prev, 14, 4353-8.

Honma N, Horii R, Iwase T, et al (2015). Ki-67 evaluation at the hottest spot predicts clinical outcome of patients with hormone receptor-positive/HER2-negative breast cancer treated with adjuvant tamoxifen monotherapy. Breast Cancer, 22, 71-8.

Ingolf JB, Russalina M, Simona M, et al (2014). Can ki-67 play a role in prediction of breast cancer patients' response to neoadjuvant chemotherapy?. Biomed Res Int, 2014, 628217.

Inwald EC, Klinkhammer-Schalke M, Hofstädter F, et al (2013). $\mathrm{Ki}-67$ is a prognostic parameter in breast cancer patients: results of a large population-based cohort of a cancer registry. Breast Cancer Res Treat, 139, 539-52.

Lakhani SR, Ellis IO, Schnitt SJ, et al (2012). WHO classification of tumours of the breast. 4ème Ed. IARC: Lyon.

Nishimura R, Osako T, Nishiyama Y, et al (2014). Prognostic significance of Ki-67 index value at the primary breast tumor in recurrent breast cancer. Mol Clin Oncol, 2, 1062-68.

Park BW, Park S, Park HS, et al (2012). Cyclooxygenase-2 expression in proliferative $\mathrm{Ki}-67$-positive breast cancers is associated with poor outcomes. Breast Cancer Res Treat, 133, 741-51.

Perou CM, Sørlie T, Eisen MB, et al (2000). Molecular portraits of human breast tumours. Nature, 406, 747-52.

Pinder SE, Wencyk P, Sibbering DM, et al (1995). Assessment of the new proliferation marker MIB1 in breast carcinoma using image analysis: associations with other prognostic factors and survival. Br J Cancer, 71, 146-9.

Polley MY, Leung SC, McShane LM, et al (2013). International Ki67 in breast cancer working group of the Breast International Group and North American Breast Cancer Group. An international Ki67 reproducibility study. J Natl Cancer Inst, 105, 1897-906.

Reyal F, Hajage D, Savignoni A, et al (2013). Long-term prognostic performance of Ki67 rate in early stage, pT1-pT2, pN0, invasive breast carcinoma. PLoS One, 8, e55901.

Sørlie T, Perou CM, Tibshirani R, et al (2001). Gene expression 
patterns of breast carcinomas distinguish tumor subclasses with clinical implications. Proc Natl Acad Sci US A, 98, 10869-74.

Stuart-Harris R, Caldas C, Pinder SE, et al (2008). Proliferation markers and survival in early breast cancer: a systematic review and meta-analysis of 85 studies in 32,825 patients. Breast $J$, 17, 323-34.

Sun J, Chen C, Wei W, et al (2015). Associations and indications of Ki67 expression with clinicopathological parameters and molecular subtypes in invasive breast cancer: A population-based study. Oncol Lett, 10, 1741-8.

Tashima R, Nishimura R, Osako T, et al (2015). Evaluation of an optimal cut-off Point for the Ki-67 index as a prognostic factor in primary breast cancer: A retrospective study. PLoS One, 10, e 0119565.

Wang B, Wang X, Wang J, et al (2014). Expression of Ki67 and clinicopathological features in breast cancer. Zhonghua Zhong Liu Za Zhi, 36, 273-5.

Wolff AC, Hammond ME, Hicks DG, et al (2013). American Society of Clinical Oncology/College of American Pathologists. Recommendations for human epidermal growth factor receptor 2 testing in breast cancer: American Society of Clinical Oncology/College of American Pathologists clinical practice guideline update. J Clin Oncol, 31, 3997-4013

\section{c) (i) (9)}

This work is licensed under a Creative Commons AttributionNon Commercial 4.0 International License. 